\title{
Selbstbestimmtes Sterben als Herausforderung an die Medizin
}

\section{Roland Kunza, Heinz Rüegger ${ }^{b}$}

a Dr. med. Roland Kunz, Facharzt für Allgemeine Innere Medizin, Schwerpunkttitel Geriatrie und Palliative Care; Chefarzt der Universitären Klinik für Akutgeriatrie im Stadtspital Waid, Zürich; Dozent für Palliative Care an der medizinischen Fakultät der Universität Zürich; ${ }^{\text {b }}$ Dr. theol. Heinz Rüegger MAE, Theologe/Ethiker/Gerontologe, Wissenschaftlicher Mitarbeiter im Institut Neumünster, Zollikerberg; assoziiertes Mitglied des Zentrums für Gerontologie der Universität Zürich

\section{Sterben im Zeichen von therapeutischen Entscheidungen}

Sterben verändert sich im Verlauf der Zeit. Es verändert sich insbesondere im Blick auf das immer grösser werdende Arsenal an medizinischen Möglichkeiten zur Lebensverlängerung. Neue minimalinvasive Eingriffe führen zu einem steten Hinausschieben der Grenzen des Machbaren. Das führt einerseits dazu, dass der Tod immer weniger plötzlich und unvermutet in ein menschliches Leben einbricht; immer häufiger ereignet sich das Sterben im Kontext einer länger sich hinziehenden Multimorbidität im hohen Alter. Andererseits stellt sich in dieser Lebensphase zunehmend die Frage, bis wann es sinnvoll und wünschbar ist, unter Ausschöpfung der heutigen medizinischen Möglichkeiten gegen den Tod anzukämpfen und das Sterben hinauszuzögern, und ab wann es angezeigt ist, den Prozess des Sterbens zuzulassen und den Tod als unausweichliches Ende jeden menschlichen Lebens zu akzeptieren. Hier drängen sich im therapeutischen Bereich in einem Mass sog. end-of-life decisions im Sinne von Passiver Sterbehilfe (Verzicht auf lebensverlängernde Massnahmen) auf, wie das früher nicht der Fall war, und solche Lebensende-Entscheidungen bestimmen heute die Sterbeverläufe massgeblich mit, wie neuste Forschungsberichte aus dem NFP-67-Programm hervorheben [1]. Waren Sterben und Tod früher Inbegriff eines fremd verfügten Schicksals, nehmen sie

\section{Zusammenfassung}

Sterben wird immer mehr von medizinischen Entscheidungen, v.a. im Blick auf den Einsatz von oder Verzicht auf lebensverlängernde Massnahmen, abhängig. Solche medical end-oflife decisions müssen letztlich vom betroffenen Patienten selbst, im Falle von dessen Urteilsunfähigkeit von dessen gesetzlich autorisierter Stellvertretung nach Art. 378 ZGB gefällt werden. Sie liegen nicht im Zuständigkeitsbereich der Ärzteschaft. Diese ist dafür umso mehr gefordert, die Betroffenen so aufzuklären und zu betreuen, dass diese befähigt werden, entsprechende Entscheide selbstbestimmt zu fällen. Das wiederum setzt eine ärztliche Gesprächskultur voraus, in der offen, sachlich und zugleich empathisch über Optionen des Sterbens unter den real existierenden Bedingungen unseres heutigen Gesundheitswesens geredet werden kann. Eine solche Kultur ist derzeit noch ein Desiderat, auf dessen Dringlichkeit dieser Artikel hinweisen will. heute zunehmend Züge eines selbst zu bestimmenden «Machsals» (O. Marquard) an - auch bei Menschen, die nie auf die Idee kämen, für sich einen Suizid in Erwägung zu ziehen. Jüngste Studien von G. Bosshard et al. haben gezeigt, dass in der Schweiz im Jahre 2013 in $58,7 \%$ der medizinisch begleiteten Todesfälle dem Sterben medizinische end-of-life decisions vorausgingen [2]

\section{Selbstbestimmtes Sterben als neues Paradigma des Sterbens}

Solche Entscheidungen fallen - schon gar seit dem Anfang 2013 erfolgten Inkrafttreten des neuen Erwachsenenschutzrechtes - ethisch und juristisch gesehen nicht mehr in den Zuständigkeitsbereich der behandelnden Ärztin. Sie stehen allein dem betroffenen $\mathrm{Pa}$ tienten selbst zu, oder falls er aufgrund einer vorliegenden Urteilsunfähigkeit dazu nicht mehr in der Lage sein sollte, der rechtlich autorisierten Vertretungsperson gemäss der Kaskade in Art. 378 ZGB. Diese jedoch hat sich streng nach dem mutmasslichen Willen der Person zu richten, die sie zu vertreten hat. Man kann es drehen und wenden, wie man will: In der Mehrzahl der Fälle stirbt man heute in der Schweiz erst, nachdem entschieden worden ist, das Sterben zuzulassen und nicht länger hinauszuschieben, und dieser Entscheid muss sich am aktuellen oder mutmasslichen Willen der betroffenen Patientin orientieren. Sie soll das letzte Wort zu sagen haben; schliesslich geht es um ihr Leben oder Sterben. Und da ist das medizin-ethische Prinzip der Patientenautonomie zentral.

Oder anders gesagt: Sterben richtet sich heute mehrheitlich nach dem Paradigma der Selbstbestimmung. Selbstbestimmtes Sterben ist bei uns zum Normalfall des Sterbens geworden. Was seit Jahrzehnten in der westlichen Welt mit dem Schlagwort des «Rechts auf den eigenen Tod» eingefordert wurde, hat sich inzwischen zu einer zugemuteten Forderung an die Sterbenden entwickelt: zu sagen, ob sie in einer bestimmten Situation allenfalls sterben oder weiterleben wollen. Wir sind heute gleichsam zur Freiheit selbstbestimmten Sterbens gezwungen, 
völlig unabhängig von den zahlenmässig nicht ins Gewicht fallenden 1,2\% der Todesfälle (2014), die auf das Konto von assistierten Suiziden à la EXIT gehen.

Wie komplex und für alle Beteiligten anspruchsvoll die sich daraus ergebende Situation sich darstellt, hat die Juristin Regina E. Aebi-Müller in dem von ihr im Rahmen des NFP 67 geleiteten Projekts «Selbstbestimmung am Lebensende im Schweizer Recht: Eine kritische Auseinandersetzung mit der rechtlichen Pflicht, selber entscheiden zu müssen» aufgezeigt. Sie weist darauf hin, dass die Rechtswissenschaften dem Patienten in jüngster Zeit zunehmend Entscheidungsverantwortung überbürdeten. «Es geht bei der Autonomie (aber) nicht nur um Gewährung von Gestaltungsspielraum, sondern auch um Verantwortung, die dem Betroffenen überbunden wird. Diese kann für den Patienten am Lebensende eine Zumutung sein. Autonomie am Lebensende darf nicht eingefordert und abgerufen werden. Vielmehr kann es nur darum gehen, Rahmenbedingungen zu schaffen, damit sie sich möglichst wirksam entfalten kann [...] Die zu starke Betonung der Patientenautonomie läuft Gefahr, sich gegen den Betroffenen zu wenden" [3].

Sterben ist zu einem Gegenstand therapeutischer Entscheidungen geworden. Oder mit den Worten des Soziologen Reimer Gronemeyer: «Das ist das Neue: Sterben und Tod sind für uns moderne Menschen zum 'Problem' geworden. Der Tod kommt nicht mehr, sondern er wird zur letzten Gestaltungsaufgabe des Menschen.» Und weiter: «Sterben wird planbar - das ist ein Grundzug der modernen Gesellschaft. Ebenso wie am Anfang des Lebens wird auch am Ende des Lebens nichts dem Zufall überlassen" [4]. Das heisst: Wir haben es heute im Blick auf selbstbestimmtes Sterben mit einem kulturgeschichtlich neuen Paradigma des Sterbens zu tun - weit über das zahlenmässig begrenzte Phänomen assistierter Suizide hinaus. Damit angemessen umzugehen, ist für das sterbende Individuum wie für die den Sterbeprozess begleitenden Angehörigen und Professionellen noch weithin ungewohnt und anspruchsvoll.

\section{Selbstbestimmung zwischen Freiheit und Überforderung}

Die beschriebene Situation bringt für den Einzelnen unzweifelhaft ein Mehr an Selbstbestimmung mit sich, allerdings zugleich ein Mehr an Selbstverantwortung. Es findet so etwas wie eine Responsibilisierung oder Moralisierung des Sterbens statt. Um noch einmal Reimer Gronemeyer zu zitieren: «Solange der Tod 'kam', musste sich keiner rechtfertigen: Es bedurfte einer solchen Debatte nicht. Das moderne Subjekt hat sich in die fatale Lage gebracht, dass es nun selbst sein Sterben und seinen Tod zu verantworten hat» [5]. Und das kann Sterbende angesichts der völlig normalen menschlichen Ambivalenz gegenüber dem Sterben überfordern. Diese Einsicht stimmt auch mit Erkenntnissen des Nationalen Forschungsprogramms «Lebensende» (NFP 67) überein: «Der Tod ... wird immer mehr zu einer Folge individueller Entscheide: Wie, wann und wo will ich sterben? Diese Fragen zu stellen und zu entscheiden, bringt zwar einen Freiheitsgewinn, aber auch eine Verantwortung mit sich, die in Überforderung münden kann» [6]. Auch Angehörige, die stellvertretend Entscheide zu fällen haben, tun sich mitunter schwer damit. Die Zürcher Medizinethikerin Tanja Krones geht davon aus, dass bis zu einem Drittel der für solche Stellvertretungsentscheide verantwortlichen Angehörigen traumatisiert werden [7].

Dafür, dass Betroffene die heute geforderte Selbstbestimmung über Leben und Sterben wahrnehmen können, sie als Ausdruck von Freiheit und nicht als Überforderung erleben, kommt den behandelnden Ärztinnen und Ärzten eine grosse Verantwortung zu.

\section{Herausforderungen an die ärztliche Praxis}

\section{Gesprächskultur}

Diese Verantwortung liegt vor allem im Entwickeln einer offenen Gesprächskultur, insbesondere in Spitälern und Heimen. Einer Gesprächskultur, die das Sterben nicht tabuisiert und peinlich umgeht, sondern selbstverständlich zur Sprache bringt. Christian Kind, Präsident der Subkommission, die im Auftrag der Zentralen Ethikkommission der SAMW die zurzeit in Vernehmlassung befindlichen neuen medizin-ethischen Richtlinien zum «Umgang mit Sterben und Tod» [8] erarbeitet hat, weist auf eine unter der Ärzteschaft verbreitete Sprachlosigkeit hin, weil «sowohl gegenüber der Auseinandersetzung mit der eigenen Sterblichkeit als auch gegenüber der Konfrontation mit kurativ-therapeutischer Ohnmacht meist grosse innere Widerstände bestehen» [9]. Und Daniel Scheidegger, Präsident der SAMW, gibt zu bedenken: «Es mag sein, dass Sterben und Tod in der öffentlichen Diskussion kein Tabuthema mehr sind; in der Medizin sind sie es leider immer noch. Wir als medizinische Fachpersonen werden für den Umgang mit Sterbenden ... nicht vorbereitet, weder in der Ausbildung noch in der Weiterbildung» [10]. Hier besteht akuter Handlungsbedarf. Die neuen SAMW-Richtlinien verstehen sich als ein Impuls in dieser Richtung, dem weitere folgen müssen, weil in Fragen von Sterben und Tod «das Gespräch mit dem Patienten herausragende Bedeutung hat» [11]. 


\section{Anerkennung der Grenzen der Medizin}

Bereits in der Präambel der medizin-ethischen Richtlinien «Palliative Care» der SAMW steht: «Ihr Ziel ist es, zu einer Haltung zu ermutigen, welche die Grenzen der Medizin anerkennt und sich dem Sterben des Patienten und dem häufig anklingenden Gefühl der Hilflosigkeit stellt» [12]. Das deckt sich mit dem fünften Ziel zum Thema Rettung und Erhaltung von Leben im Positionspapier der SAMW zu den Zielen und Aufgaben der Medizin im 21. Jahrhundert: «Obwohl sich die Medizin spontan auf die Seite des Lebens stellt und demzufolge den Tod verhindern möchte, sollte diese Bemühung immer auf einer grundsätzlichen Akzeptanz der Sterblichkeit und des Todes beruhen. Entscheide über Lebensrettung und Lebenserhaltung bedürfen aufgrund der immensen technischen Fortschritte besonderer Sorgfalt; der Grundsatz 'Erlaubt ist, was man kann' soll nicht gelten. Die Beachtung und Wahrung des Selbstbestimmungsrechtes des Patienten - soweit er dazu im Stande ist - ist hier besonders wichtig» [13]. Diese Haltung der Akzeptanz, dass Sterben kein Scheitern der ärztlichen Kunst ist, sondern ganz und gar zum Leben gehört, ist die Voraussetzung für die Gespräche mit dem Patienten und den Angehörigen. Sie muss bereits in der Weiterbildung der kommenden Ärztegeneration verankert werden, indem sie von den Kaderärzten in den Kliniken vorgelebt wird. Es geht um das Ernstnehmen der Selbstbestimmung des Patienten bzw. dessen Stellvertretung in medizinischen Angelegenheiten nach Art. 378 ZGB (keine unerwünschte Über-Therapie).

\section{Aufklärung darüber, wie man heute sterben kann}

Zur Patienten-Aufklärung gehört auch die Information, woran der Betroffene aufgrund seiner Grundkrankheiten wie sterben kann, welche möglichen Sterbeprozesse u.U. leichter, welche belastender sind und welche Möglichkeiten die Palliativmedizin hat. «Eine verständliche und wiederholte, stufenweise Aufklärung versetzt den Patienten in die Lage, realistische Erwartungen zu entwickeln, und ermöglicht eine eigenständige Willensbildung und Entscheidung. Grundvoraussetzungen dazu sind ... die Bereitschaft, Möglichkeiten und Grenzen der kurativen wie der palliativen Behandlung offen zu legen" [14]. Diese Beratung erwarten die Menschen heute von ihrem Arzt, und sie möchten über die verschiedenen Möglichkeiten des Sterbens aufgeklärt werden [15]; nur so können sie autonom entscheiden. End-of-life decisions sind Prozesse und nicht Folge eines einzelnen Gesprächs auf der Visite. Sie erfordern die Bereitschaft, mit Patienten deren Ambivalenz gegenüber solchen Entscheidungen auszuhal- ten und geduldig immer wieder über die anstehenden Entscheidungen zu sprechen [16].

\section{Forschung}

Bisher misst die Forschung den Erfolg einer medizinischen Massnahme immer noch einseitig am Endpunkt der Lebensverlängerung. Sie setzt damit voraus, dass Lebensverlängerung auch für die betroffene Patientin immer das wichtigste Ziel ist. Erst wenige Studien haben sich mit dem Goal of Care multimorbider alter Menschen auseinandergesetzt. Und sie haben gezeigt, dass ältere Menschen lieber Prioritäten, Werthaltungen und Ziele sowie Grenzen für ihr verbleibendes Leben formulieren möchten als explizite Entscheidungen $\mathrm{zu}$ medizinischen Weichenstellungen zu treffen [17]. Diese überlassen sie lieber ihren Angehörigen und dem Behandlungsteam. Wir müssen somit in der Forschung neue Endpunkte definieren und davon wegkommen, dass ein späterer Tod mit grosser Wahrscheinlichkeit ein besserer Tod sei [18].

\section{Literatur}

1 Leitungsgruppe des NFP 67 Lebensende (Hg.). Synthesebericht NFP 67 Lebensende. Bern: Schweizerischer Nationalfonds 2017; p. 5.20

2 Bosshard G, Hurst SA, Puhan MA. Medizinische Entscheidungen am Lebensende sind häufig. Swiss Medical Forum - Schweizerisches Medizin-Forum. 2016;16(42):896-8.

3 Aebi-Müller RE, Dörr BS, Haussener S, Waldenmeyer C. Selbstbestimmung am Lebensende im Schweizer Recht: Eine kritische Auseinandersetzung mit der rechtlichen Pflicht, selber entscheiden zu müssen (Lay Summary). Luzern, 11.4.2017, p. 4f.

4 Gronemeyer R. Sterben in Deutschland. Wie wir dem Tod wieder einen Platz in unserem Leben einräumen können. Frankfurt a.M.: Fischer 2007;37.181

5 Gronemeyer R. 2007; p. 177

6 Leitungsgruppe des NFP 67 Lebensende (Hg.). Synthesebericht NFP 67 Lebensende. Bern: Schweizerischer Nationalfonds 2017; p. 9.

7 Krones T. Familienangehörige als vertretungsberechtigte Personen. Jusletter 25.1.2016; Rz 8.

8 Die am 16.11.2017 in Vernehmlassung gegebene Version der neuen Richtlinien findet sich unter https://www.samw.ch/de/Ethik/ Sterben-und-Tod/Richtlinien-Sterben-Tod.html

9 Kind C. Umgang mit Sterben und Tod: die revidierten medizinethischen Richtlinien im Fokus. SAMW-Bulletin 04/2017, p. 1-4, dort p. 1.

10 Scheidegger D. Das Sterben gehört zum Menschen - und zur Medizin. SAMW-Bulletin 04/2017, p. 2

11 So die Präambel der neuen SAMW-Richtlinie.

12 SAMW. Medizin-ethische Richtlinien und Empfehlungen (zu) Palliative Care; 2006/2013. Bern 2017 (9. Aufl.); p. 5.

13 SAMW. Positionspapier: Ziele und Aufgaben der Medizin im 21. Jahrhundert (2004); p. 33.

14 SAMW. Medizin-ethische Richtlinien und Empfehlungen (zu) Palliative Care; 2006/2013. Bern 2017 (9. Aufl.); p. 10

15 LINK Institut. Letzter Lebensabschnitt. Was erwartet die Bevölkerung vom Arzt? 2016 (URL: https://www.exit.ch/ fileadmin/user_upload/files/Studie_LINK_Institut_Erwartungen_an_Aerzte_2016.pdf).

16 Ohnsorge K, Gudat Keller HR, Widdershoven GAM, Rehmann Sutter C. Ambivalence at the end of life. How to understand patients' wishes ethically. Nursing ethics 2012 (19); pp. 629-41.

17 Romo RD et al. Sense of control in end-of-life decision-making. J Am Geriatr Soc. 2017;65:E70-E75.

18 Callahan D. The Troubled Dream of Life. In Search of a Peaceful Death. Washington: Georgetown University 2000; p. 187-219. 